\title{
1. Verrechnungspreise im Spannungsfeld von Controlling und Steuern
}

"Transfer pricing is not an exact science ${ }^{1}{ }^{1}$ Dennoch stehen Verrechnungspreise, die Wertansätze zur Überführung und Verrechnung betriebsinterner Leistungsflüsse darstellen, ${ }^{2}$ aktuell vermehrt im Fokus von Betriebsprüfungen. ${ }^{3}$ Damit stellen sie ein beträchtliches Risiko für international agierende Unternehmen dar. ${ }^{4}$ Schätzungen zufolge findet heutzutage ca. $70 \%$ des Welthandels zwischen international verbundenen Unternehmen statt. ${ }^{5}$ Hierbei dient der Verrechnungspreis zur steuerlichen Erfolgsermittlung international ansässiger Verbundunternehmen und wirkt auf die Steuereinnahmen der beteiligten Staaten. ${ }^{6}$ Der Verrechnungspreis sollte daher für die interne Leistung aus Besteuerungssicht einen verursachungsgerechten Anteil des Gesamtgewinns beinhalten. ${ }^{7}$ Hierfür wurden international vermehrt steuerliche Regelungen erlassen, die die Bestimmung und Korrektur von Verrechnungspreisen regulieren. ${ }^{8}$ Im Controlling werden Verrechnungspreise vordergründig als übergreifendes Koordinationsinstrument dezentraler Einheiten verwendet. ${ }^{9}$ Optimale Wirkungen bei der gleichzeitigen Verfolgung mehrerer, meist kontroverser Zielsetzungen mit nur einem Verrechnungspreis können regelmäßig nicht erzielt werden. ${ }^{10}$ Dabei steht besonders ein Verrechnungspreis, der einerseits zur Koordination und andererseits zur rechtskonformen Besteuerung dienen soll, im Spannungsfeld zwischen dem Controlling und der Steuerabteilung. ${ }^{11}$ Dies führt in der Unternehmenspraxis häufig zur Verfolgung nur einer Zielsetzung mit Verrechnungspreisen. Zukünftig wird jedoch auf eine konfliktfreie Verbindung beider Zielsetzungen in einem Verrechnungspreissystem abgezielt. Dies zeigt die Notwendigkeit dieser Simulationsstudie, die die Wirkungen der Zielsetzung der Koordination und der steuerlichen Erfolgsermittlung in Form eines Verrechnungspreises, der als steuerinduzierter Lenkpreis bezeichnet wird, analysiert. Hieraus ergeben sich Erkenntnisse für die zukünftige Gestaltung von steuerinduzierten Lenkpreisen in Unternehmen zur Erzielung gewünschter Wirkungen.

\footnotetext{
${ }^{1}$ OECD [Leitlinien] Ziffer 1.13, 3.55, 4.8 und 8.3

${ }^{2}$ vgl. Schweitzer/Baumeister [Unternehmen] 998

${ }^{3}$ vgl. Baumhoff [Verrechnungspreise] 8, Bittner/Dawid/Metzner [Betriebsprüfung] $199 \mathrm{f}$. , Ditz/Bärsch/Kluge [Praxis] 821, Ditz etal. [Spannungsfeld] 2596 f., Dorner [Fremdvergleichsgrundsatz] 2, Schneider [Verrechnungspreisrisiken] 119, Wehnert/Waldens/Sprenger [Verrechnungspreise] 2901 und Wilmanns [Verrechnungspreise] $34 \mathrm{f}$.

${ }^{4}$ vgl. Ullmann/Trede [Verrechnungspreise] 330

${ }^{5}$ vgl. Baumhoff [Methoden] 378,Crüger/Ritter [Steuerung] 497 und Wehnert/Waldens/Sprenger [Verrechnungspreise] 2901

${ }^{6}$ vgl. Baumhoff [Methoden] 380, Bittner/Heidecke [Verrechnungspreise] 119, Dorner [Fremdvergleichsgrundsatz] 2 und Weber/Schäffer [Controlling] 213

7 vgl. Baumhoff [Verrechnungspreise] 6 ff., Spengel/Oestreicher [Steuerbemessung] 780 und Wassermeyer [Hintergrund] 8

${ }^{8} \mathrm{vgl}$. Baumhoff [Methoden] 381, Bittner/Heidecke [Verrechnungspreise] 120

${ }^{9}$ vgl. Horváth/Gleich/Seiter [Controlling] 302

${ }^{10}$ vgl. Dürr/Göx [Transfer] 28 ff., Weber/Schäffer [Controlling] 215 und Schweitzer/Baumeister [Unternehmen] 1004

${ }^{11} \mathrm{vgl}$. auch fortfolgend Ditz etal. [Spannungsfeld] $2593 \mathrm{ff}$.
} 